\title{
Effects of preparation methods of mixed calcium and zinc thermal stabilizers derived from dimer fatty acid and tung-oil based $\mathrm{C} 22$ triacid on properties of PVC
}

\author{
Mei Li ${ }^{1,2}$, Mei Wang ${ }^{1}$, Shouhai $\mathrm{Li}^{1,2}$, Kun Huang ${ }^{1}$, Wei Mao ${ }^{1}$, Jianling Xia ${ }^{1,2^{*}}$ \\ ${ }^{1}$ Institute of Chemical Industry of Forestry Products, CAF; Jiangsu Province Biomass Energy and Materials Laboratory; \\ National Engineering Lab for Biomass Chemical Utilization; Key Lab on Forest Chemical Engineering, SFA, Nanjing \\ 210042, Jiangsu Province, P.R. China \\ ${ }^{2}$ Research Institute of Forestry New Technology, CAF, Beijing 100091, P.R. China \\ *corresponding author: e-mail: xiajianling@126.com
}

\begin{abstract}
Calcium and zinc salts of dimer fatty acids (DFA-Ca and DFA-Zn) were synthesized using direct neutralization and metathesis technologies, respectively. The adduct of maleic anhydride and methyl eleostearate (MAME) was also converted to the corresponding zinc soap (C22TA-Zn) and calcium soap (C22TA-Ca) by the two different synthetic routes. Mixed $\mathrm{Ca} / \mathrm{Zn}$ salts between DFA-Ca and DFA-Zn, and between C22TA-Zn and C22TA-Ca were used as thermal stabilizers for poly(vinyl chloride) (PVC). The PVC thermal stability was determined using Congo red test, discoloration test, torque rheological analysis and TGA. Dynamic mechanical properties were also tested. Results indicated that the DFA-Ca/DFA-Zn thermal stabilizer from direct neutralization technology was found to be superior to that of the metathesis product. The C22TA-Ca/C22TA-Zn thermal stabilizer from direct neutralization method had overall superior thermal stability, and displayed modulus and glass transition comparable to that of metathesis product. Direct neutralization method was more excellent and convenient than metathesis technology.
\end{abstract}

Keywords: poly(vinyl chloride), mixed calcium and zinc thermal stabilizer, synthesis technology, thermal stabilization.

\section{INTRODUCTION}

Poly (vinyl chloride) (PVC) is inherent to auto-catalytic dehydrochrination ${ }^{1-2}$ which could result in severe degradation of the polymer and potential harm to the processing equipment. Thermal stabilizers are necessary to be in the formulation to prevent the undesirable degradation of PVC during process ${ }^{3-5}$. A variety of PVC thermal stabilizers are available for various applications, including epoxidized sunflower oil ${ }^{5}$, layered and intercalated hydrotalcite-like materials ${ }^{6-7}$, metal soaps (calcium, zinc, lanthanum, aluminium, etc. $)^{8-12}$, organotin compounds ${ }^{13}$ and lead salts ${ }^{14}$. Though lead salts and organotin compounds are very efficient in stabilizing PVC during processing, the former are highly toxic while the latter are expensive. Therefore, industry gradually shifts its focus to the low-cost or non-toxic and more economical calcium and zinc carboxylates for thermal stabilizers.

PVC processing stabilization can be gauged by short-term stability (or early color) and long-term stability (often called point-to-black) ${ }^{15}$. Another definition of short-term stability is "off-the-mill" color, and the long-term stability is the point at which the degradation either has made the compound no longer reclaimable or begins to endanger the processing equipment ${ }^{15}$. Calcium carboxylate salts provide long-term thermal stability for PVC by absorbing $\mathrm{HCl}$. In contrast, zinc carboxylate salts effectively inhibit discoloration by substituting labile chlorine atoms in PVC chains, but do not provide long-term stability ${ }^{16-18}$. However, the mixed $\mathrm{Ca} / \mathrm{Zn}$ thermal stabilizers at an appropriate ratio exhibit synergistic effects with both acceptable initial color and long-term stability for PVC products, 19-20.

Calcium and zinc salts of carboxylic acids (also called soaps) derived from dimer fatty acids (DFA), tung-maleic anhydride, and so on can be prepared by either direct neutralization process ${ }^{21-23}$ or metathesis process ${ }^{24-26}$. For example, calcium sebacate and zinc sebacate were synthesized directly by reacting calcium hydroxide and zinc hydroxide aqueous solution with sebacic acid, respectively, and the precipitated salts (soaps) were collected by filtration and then washed with water ${ }^{21}$. The zinc and calcuim salts of glutaric acid - a dicarboxylic acid, were prepared similarly by reacting glutaric acid with zinc oxide $(\mathrm{ZnO})$ and calcium oxide $(\mathrm{CaO})$ in toluene, respectively ${ }^{22}$. The zinc and calcium salts of dicarboxylic acid can also be prepared by a metathesis process, i.e., neutralization with $\mathrm{NaOH}$ follwed by salt metathesis reaction ${ }^{24}$. However, the metathesis process with water as solvent and mild reaction conditions resulted in a lot of waste water and products of low purity and light color ${ }^{27}$. In contrast, the direct neutralization process has the advantages of simple process, short production cycle and high yield, but it tends to give the product with a darker color ${ }^{28}$. On the other hand, the difference in stabilizing effects and thermal degradation kinetics between the two mixed $\mathrm{Ca} /$ $\mathrm{Zn}$ thermal stabilizers prepared by direct neutralization and metathesis process is still unknown.

In this work, calcium and zinc salts of dimer fatty acids (DFA) and the adduct of maleic anhydride and methyl eleostearate (MAME) were introduced as PVC thermal stabilizers. DFA and MAME were converted to calcuim and zinc salts by direct neutralization and metathesis, respectively. Thermal stabilizing effects of the mixed $\mathrm{Ca} / \mathrm{Zn}$ salts prepared from both processes were investigated and compared. In addition-dynamic mechanical properties of the stabilized PVC compounds were also studied. 


\section{EXPERIMENTAL}

\section{Material}

The adduct of maleic anhydride and methyl eleostearate (MAME) (acid value, $269.0 \mathrm{mg} \mathrm{KOH} / \mathrm{g}$; saponification value, $360 \pm 10 \mathrm{mg} \mathrm{KOH} / \mathrm{g}$, color $(\mathrm{Fe}-\mathrm{Co}) \leq 15)$ was obtained from the Institute of Chemical Industry of Forestry Products. The product was a mixture of maleated methyl esters of various fatty acids with MAME as the major component. Dimer fatty acid (dimers $\geq 95 \%$, acid value: $204.4 \mathrm{mg} \mathrm{KOH} / \mathrm{g}$ ) was obtained from the Shanghai Guxiang Chemical Company. PVC (DG-1000K) was purchased from Tianjin Dagu Chemical Co., Ltd, China. Commercial calcium and zinc stearates $\left(\mathrm{CaSt}_{2}\right.$, calcium content: $6.59 \% ; \mathrm{ZnSt}_{2}$, zinc content: $10.28 \%$ ) were purchased from the Xilong Chemical company, China. The Bis (2-ethylhexyl) terephthalate (DOTP) was obtained from Acros Organics. Dioctyl phthalate (DOP) and epoxidized soybean oil (ESO) were obtained from Qingzhou Shuntong Plastic Additive Factory, China. Commercial $\beta$-diketone (DK-melting point: $70.7^{\circ} \mathrm{C}$ ) was obtained from Changzhou JiaRenWo Chemical Company.

\section{SYNTHESIS}

Preparation of zinc and calcium soaps of dimer fatty $\operatorname{acid}($ DFA)

Synthesis of zinc and calcium soaps of DFA by direct neutralization

Calcium and zinc soaps were synthesized by reacting DFA with $\mathrm{Ca}(\mathrm{OH})_{2}$ and $\mathrm{ZnO}$, respectively. $\mathrm{DFA}(0.1 \mathrm{~mol})$ was dissolved in 1,4-dioxane $(200 \mathrm{~mL})$, the mixture was introduced into a 1-L four-necked round-bottom flask equipped with a mechanical stirrer, a thermometer and a reflux condenser. The mixture was then heated to $80^{\circ} \mathrm{C}$ before adding $\mathrm{Ca}(\mathrm{OH})_{2}(0.099 \mathrm{~mol})$ and $\mathrm{ZnO}$ $(0.099 \mathrm{~mol})$. The final mixture was heated to $120^{\circ} \mathrm{C}$; the reaction was continued for $3 \mathrm{~h}$. Finally, the solvents were removed using vacuum distillation. Hereafter, the single-step DFA calcium and zinc salts are referred to as DFA-Ca-I and DFA-Zn-I.

\section{Synthesis of zinc and calcium soaps of DFA by metathesis}

Both zinc and calcium salts of DFA were prepared in a two-step, neutralization followed by salt metathesis. Sodium soap of diacid was prepared by mixing $0.1 \mathrm{~mol}$ of diacid and $0.2 \mathrm{~mol}$ of $\mathrm{NaOH}$ using $150 \mathrm{~mL}$ acetone and $5 \mathrm{~mL}$ deionized water as solvents. The mixture was stirred vigorously at $50^{\circ} \mathrm{C}$ for $30 \mathrm{~min}$. After cooling to $-15^{\circ} \mathrm{C}$ for $24 \mathrm{~h}$, the reaction mixture was filtered and washed with acetone several times, giving sodium soap in powder form. The sodium soap was dried in a vacuum at $70^{\circ} \mathrm{C}$ for $10 \mathrm{~h}$.

In preparing the calcium soaps, 0.1 mol sodium soap was dissolved in $100 \mathrm{~mL}$ of water, and slowly added to $0.1 \mathrm{~mol}$ of $\mathrm{CaCl}_{2}$ solution $\left(0.1 \mathrm{~mol} \mathrm{CaCl}_{2}\right.$ dissolved in $100 \mathrm{~mL}$ ethanol and $100 \mathrm{~mL}$ of deionized water). The reaction was continued at $70^{\circ} \mathrm{C}$ for $3 \mathrm{~h}$. The precipitated metal soap was washed several times with deionized water, filtered, and dried in an oven under a $400 \mathrm{mg} \mathrm{Hg}$ vacuum at $70^{\circ} \mathrm{C}$ for $24 \mathrm{~h}$. Zinc soaps were prepared from zinc sulfate $\left(\mathrm{ZnSO}_{4} \cdot 7 \mathrm{H}_{2} \mathrm{O}\right)$ and diacid in a procedure similar to that of the calcium soaps. The DFA calcium and zinc salts obtained in two-step are referred to as DFA-Ca-II and DFA-Zn-II.

\section{Preparation of zinc and calcium soaps of C22TA}

Scheme 1 shows the synthesis route of C22TA-Ca and C22TA-Zn by the direct neutralization method and metathesis technology.

Synthesis of zinc and calcium soaps of C22TA by direct neutralization

1) Synthesis of C22 tricarboxylic acid (C22TA).

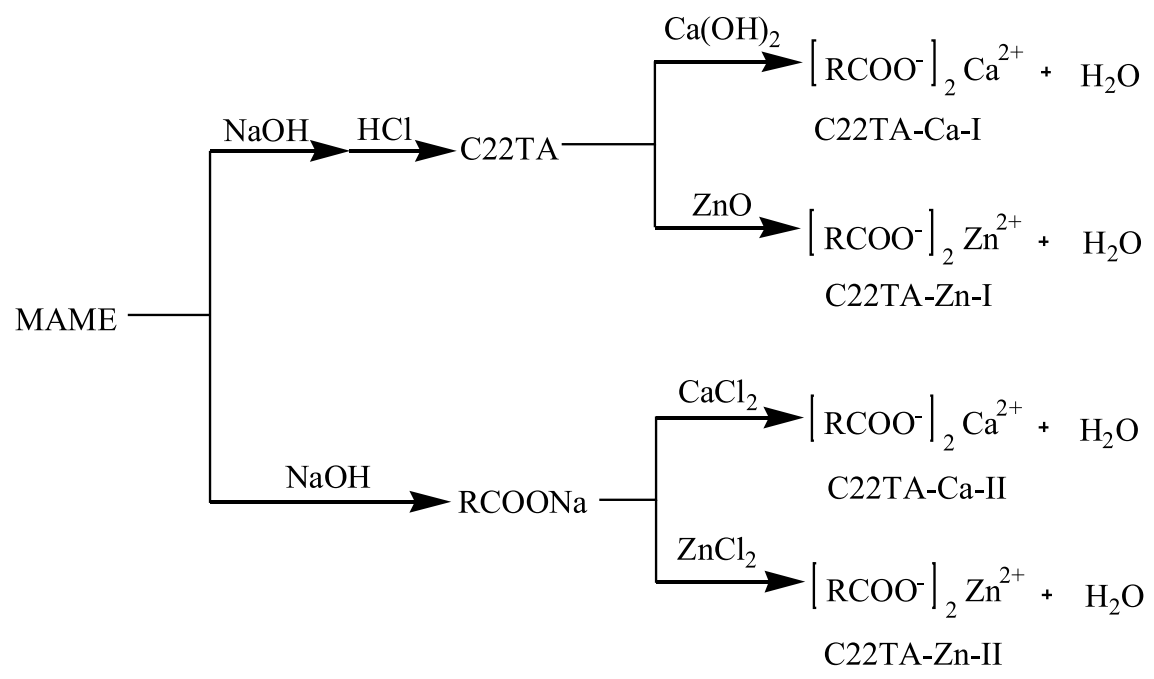

The major ingredient of MAME is shown below:

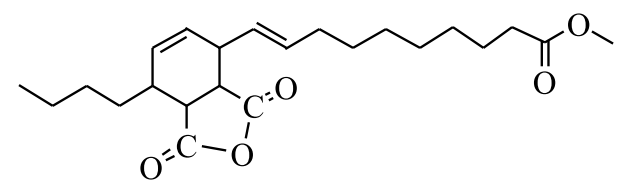

and

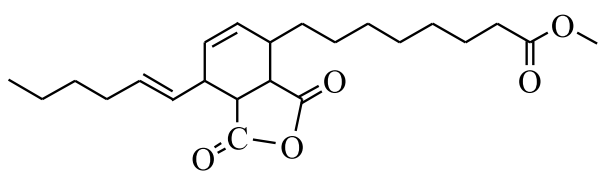

Scheme 1. Synthetic routes of direct neutralization and metathesis process of calcium and zinc salts of the C22TA tricarboxylic acid 
$\mathrm{NaOH}(46.15 \mathrm{~g})$ deionized water $(50 \mathrm{~mL})$ ethanol (100 $\mathrm{mL}$ ) were charged into a $500 \mathrm{~mL}$ four-necked round-bottom flask equipped with a mechanical stirrer, a thermometer and a reflux condenser. Next, the mixture was heated to $70^{\circ} \mathrm{C}$, and MAME $(100 \mathrm{~g})$ was slowly dropped into the flask. Reaction was continued at $70^{\circ} \mathrm{C}$ for $1.5 \mathrm{~h}$. After the reaction was cooled to room temperature, $\mathrm{HCl}$ aqueous solution was added until the $\mathrm{pH}$ value of the reaction mixture reached 2 . The mixture was stirred at $40^{\circ} \mathrm{C}$ for $30 \mathrm{~min}$ and then washed with deionized water until the $\mathrm{pH}$ value of the water was 7. After the residual water was removed using a rotary evaporator under vacuum, the product $\mathrm{C} 22$ tricarboxylic acid (C22TA) with an acid value of $379.4 \mathrm{mg} \mathrm{KOH} / \mathrm{g}$ was obtained.

2) Preparation of zinc and calcium soaps of C22TA by direct neutralization.

Calcium soap and zinc soap were synthesized by reacting C22TA with $\mathrm{Ca}(\mathrm{OH})_{2}$ and $\mathrm{ZnO}$, respectively. C22TA (0.05 mol) was dissolved in 1, 4-dioxane (200 $\mathrm{mL}$ ), and the mixture was introduced into a 1-L four-necked round-bottom flask equipped with a mechanical stirrer, a thermometer and a reflux condenser. Next, the mixture was heated to $80^{\circ} \mathrm{C}$, and $\mathrm{Ca}(\mathrm{OH})_{2}(0.0735 \mathrm{~mol})$ or $\mathrm{ZnO}(0.0735 \mathrm{~mol})$ was added. The final mixture was heated to $120^{\circ} \mathrm{C}$ and kept reacting for $3 \mathrm{~h}$. Finally, the solvent was removed by vacuum distillation. Hereafter, the C22TA calcium salt (C22TA -Ca) and C22TA zinc salt (C22TA-Zn) prepared from direct neutralization method are named C22TA-Ca-I, C22TA-Zn-I, respectively.

\section{Synthesis of zinc and calcium soaps of C22TA by me- tathesis}

The zinc soaps and calcium soaps of C22TA were prepared by sodium salt formation followed by metathesis (Scheme 1). Sodium soap of C22TA was prepared by mixing 0.019 mol MAME (7.8 g ) and $0.06 \mathrm{~mol} \mathrm{NaOH}$ $(2.4 \mathrm{~g})$ in a mixture of ethanol $(45 \mathrm{~mL})$ and water $(15 \mathrm{~mL})$. The mixture was stirred vigorously at $70^{\circ} \mathrm{C}$ for $2 \mathrm{~h}$ and then neutralized using a $6 \mathrm{M} \mathrm{HCl}$ solution until the $\mathrm{pH}$ value was $8 \sim 9$. Next, the solution of sodium soap was slowly added to a $0.029 \mathrm{~mol} \mathrm{CaCl}$ solution $(3.36$ $\mathrm{g}$ of $\mathrm{CaCl}_{2}$ with a purity of $96 \%$ in a mixutre of $50 \mathrm{~mL}$ of deionised water and $100 \mathrm{~mL}$ ethanol). The reaction was continued at $70^{\circ} \mathrm{C}$ for $2 \mathrm{~h}$. The precipitated calcium soap was washed several times with deionised water, filtered and dried in an oven under vacuum at $70^{\circ} \mathrm{C}$ for $24 \mathrm{~h}$. Zinc soap was prepared from the reaction of zinc chloride (4.03 $\mathrm{g}$ of $\mathrm{ZnCl}_{2}$ with a purity of $98 \%$ dissolved in a mixture of $50 \mathrm{~mL}$ of deionised water and $100 \mathrm{~mL}$ ethanol) and sodium soap of C22TA in a procedure similar to that for calcium soaps. Hereafter, the C22TA calcium salt (C22TA-Ca) and C22TA zinc salt (C22TA$-\mathrm{Zn}$ ) obtained from the metathesis process are named C22TA-Ca-II, C22TA-Zn-II, respectively.

\section{CHARACTERIZATION}

Fourier Transform Infrared (FTIR) analysis was performed on an IS10 spectrometer (Nicolet, America) in an attenuated total reflectance (ATR) mode. The film sample was scanned from 4.000 to $400 \mathrm{~cm}^{-1}$ for 16 times with a resolution of $4 \mathrm{~cm}^{-1}$.
The metal ( $\mathrm{Ca}$ and $\mathrm{Zn}$ ) content of the thermal stabilizers was measured using an Optima 7000 inductively coupled plasma atomic emission spectrometer (ICP-AES) (PerkinElmer, USA).

\section{Preparation of PVC films}

The formulations of all PVC compounds were shown in Table 1 and Table 3. First, PVC powder, DOP(or DOTP), thermal stbilizers and auxiliary heat stabilizers (ESO and DK) were mixed using a mechanical mixer at room temperature for $5 \mathrm{~min}$. Subsequently, the mixture was compounded in a homogeneous mixer at $165^{\circ} \mathrm{C}$ for 3 min using a Haake torque rheometer (Thermo, Germany). Films $(\sim 1.5 \mathrm{~mm}$ thick) were made using a mini hot press (model 3912 , Carver) at $\sim 175^{\circ} \mathrm{C}$ to $185^{\circ} \mathrm{C}$.

\section{THERMAL AND MECHANICAL PROPERTIES OF STABILIZED PVC}

\section{Thermal stability analysis}

Static thermal stability analysis was performed by Congo Red test according to ISO 182-1-1990. A piece of PVC film (about $2 \mathrm{~mm} \times 2 \mathrm{~mm}$ ) was placed in a closed test tube which was heated to $180^{\circ} \mathrm{C}$ until a strip of CR paper placed at the top of the tube changed color from red to blue (equivalent to $\sim \mathrm{pH} 3$ ). The time taken for the color change is referred as the stability time. The schematic diagram of the Congo red test device was described in Ref. ${ }^{13}$.

Dynamic thermal stability analysis was performed by torque rheology according to ASTM D 2538-02 as follows. PVC and thermal stabilizer were mixed in a Haake Rheometer RC90 mixing chamber at $180^{\circ} \mathrm{C}$ with a rotor speed of $30 \mathrm{rpm}$ for $60 \mathrm{~min}$. The dynamic thermal stabilizing time is defined as the time when the torque on the rotor starts to change abruptly.

The discoloration test of PVC samples stabilized by different thermal stabilizers was tested according to ISO 305:1990(E). The PVC films with thickness of $\sim 1 \mathrm{~mm}$ were cut into squares with a length of $15 \mathrm{~mm}$, and then each square was wrapped individually using aluminium-foil paper. The empty test tubes were placed in a temperature-controlled oil bath at $185^{\circ} \mathrm{C}$ for $20 \mathrm{~min}$, and then to each test tube was added one wrapped square strip. From this point, one test tube was removed from the oil bath every $10 \mathrm{~min}$. The effect of the stabilizers was evaluated by the comparisons of visual color differences of the heated PVC strips.

Thermal decomposition kinetics was studied using a Q600 TGA instrument (TA Instruments). Each sample was scanned from 30 to $400^{\circ} \mathrm{C}$ under nitrogen at flow rate of $100 \mathrm{~mL} / \mathrm{min}$ and heating rates of $5,10,15,20$ and $25^{\circ} \mathrm{C} / \mathrm{min}$. The Kissinger equation yields a simple relationship between the peak temperature on the DTG curve $\left(T_{p}\right)$ and the heating rate $(\beta)$. Kinetic parameters such as activation energy $(E, \mathrm{~kJ} / \mathrm{mol})$ and pre-exponential factor $(A)$ were evaluated using the Kissinger equation: $\ln \left(\frac{\beta_{i}}{T_{p}^{2}}\right)=\ln \frac{A_{k} R}{E_{k}}-\frac{E_{k}}{R} \frac{1}{T_{p}} \quad[i=1,2,3,4,5]$

where $R=$ Gas constant $(8.314 \mathrm{~J} / \mathrm{mol} \cdot \mathrm{K})$. Equation (1) indicates that $\ln \left(\beta_{i} / T_{p}^{2}\right)$ and $1 / T_{p}$ have a linear correlation. 
Finally, activation energy $(E)$ and pre-exponential factor $(A)$ can be calculated from the slope $(a)$ and intercept (d) on the curves of $\ln \left(\beta_{i} / T_{p}{ }^{2}\right)$ change versus $1 / T_{p}$.

$E_{\mathrm{k}}=-a \cdot R$

$\ln \left(\mathrm{A}_{\mathrm{k}}\right)=d+\ln \left(\frac{E_{\mathrm{k}}}{R}\right)$

\section{DYNAMIC MECHANICAL ANALYSIS (DMA)}

Dynamic mechanical analysis (DMA) of the sample was performed on a DMA Q800 (TA Instruments) in a dual-cantilever mode with an oscillating frequency of $1 \mathrm{~Hz}$. The temperature was swept from -80 to $80^{\circ} \mathrm{C}$ at a heating rate of $3^{\circ} \mathrm{C} / \mathrm{min}$. For each sample, duplicate tests were performed in order to ensure the reproducibility of data. $T_{\mathrm{g}}$ was determined from the maximum peak temperature of the $\tan \delta$ curve.

\section{RESULTS AND DISCUSSION}

\section{Characterization of C22TA, DFA, C22TA-Ca, C22TA-Zn, DFA-Ca and DFA-Zn}

The FTIR spectra of the zinc and calcium dicarboxylates (DFA-Zn-I, DFA-Zn-II \& DFA-Ca-I, DFA-Ca-II) are shown in Figure 1. The strong absorption peak at $3200 \sim 2500 \mathrm{~cm}^{-1}$ in the FTIR spectrum of the DFA (attributed to the hydroxy of the carboxyl group) nearly disappeared in DFA-Zn-I, DFA-Zn-II, DFA-Ca-I and DFA-Ca-II. Similarly, the absorption peak at 1710 $\mathrm{cm}^{-1}$ in the FTIR spectrum of the DFA, attributed to carbonyl, nearly disappeared in DFA-Zn-I, DFA-Zn-II, DFA-Ca-I and DFA-Ca-II. The absorption peaks at 1551, 1542,1539 and $1549 \mathrm{~cm}^{-1}$ were due to the asymmetric stretching vibration of the carboxylate. The absorption peaks at 1418, 1445, 1456 and $1457 \mathrm{~cm}^{-1}$ were due to the symmetric stretching vibration of the carboxylate. Based on the acid value of DFA, the theoretical Ca and $\mathrm{Zn}$ contents in DFA-Ca and DFA-Zn would be $6.82 \%$ and $10.62 \%$, respectively. The measured $\mathrm{Ca}$ and $\mathrm{Zn}$ contents in DFA-Ca-I and DFA-Zn-I were 5.78\% and $9.15 \%$, respectively. The measured $\mathrm{Ca}$ and $\mathrm{Zn}$ contents in DFA-Ca-II and DFA-Zn-II were $6.55 \%$ and $10.20 \%$, respectively, which suggest the conversions of the carboxylic acid groups were $96.04 \%$ and $96.01 \%$, respectively. The FTIR and ICP-AES results suggested that DFA was successfully converted to calcium and zinc soaps.

Figure 2 shows the FT-IR spectra of MAME, C22TA, C22TA-Ca and C22TA-Zn salts. The absorption peaks at $1864 \mathrm{~cm}^{-1}$ and $1781 \mathrm{~cm}^{-1}$ in the FTIR spectrum of MAME, which were attributed to the acid anhydride, disappeared in the C22TA and corresponding salt products. The absorption peak at $\sim 1550 \mathrm{~cm}^{-1}$ was due to the antisymmetric stretching vibration of carboxylate and the absorption peak at $\sim 1414 \mathrm{~cm}^{-1}$ attributed to the symmetric stretching vibration of carboxylate. The absorption peaks at $1704 \mathrm{~cm}^{-1}, 1717 \mathrm{~cm}^{-1}$ and $1736 \mathrm{~cm}^{-1}$ which were attributed to the carbonyl, almost disappeared in the spectra of the salt products. Based on the acid value and saponification value of MAME, the theoretical $\mathrm{Ca}$ and $\mathrm{Zn}$ contents in C22TA-Ca and C22TA-Zn would be $11.46 \%$ and $17.36 \%$, respectively. However, the measured $\mathrm{Ca}$ and $\mathrm{Zn}$ contents in C22TA-Ca-I and C22TA-Zn-I were $12.08 \%$ and $19.70 \%$, respectively. This

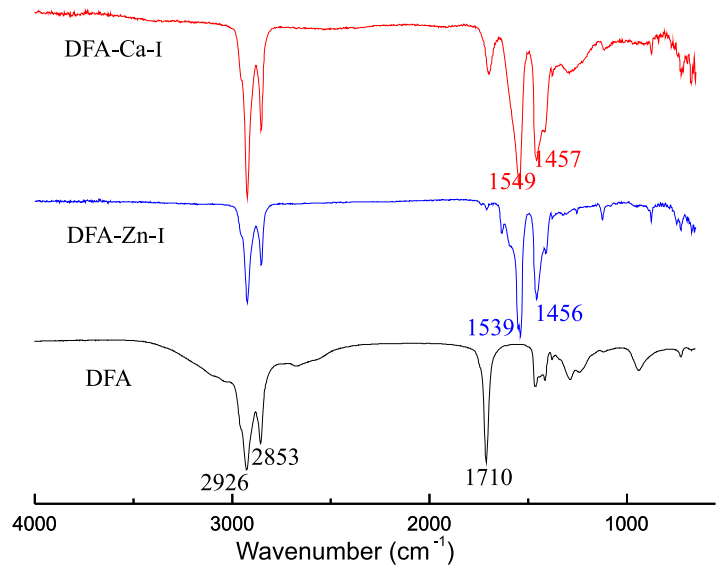

(a)

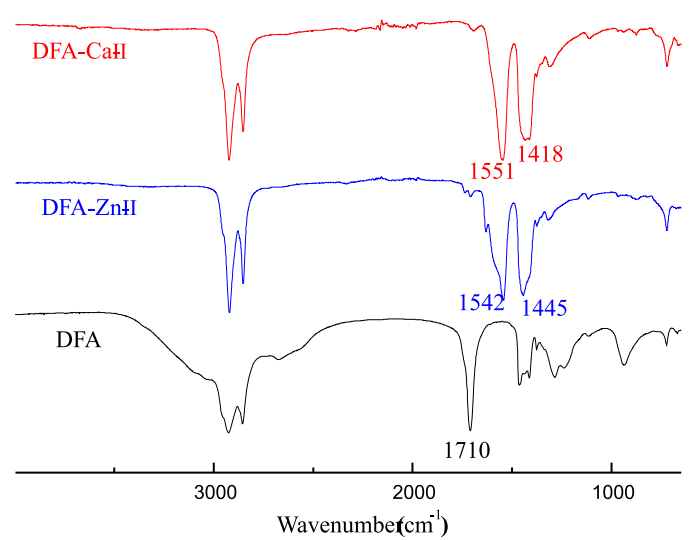

(b)

Figure 1. FTIR spectra of DFA, DFA-Ca and DFA-Zn. (a) and (b) were calcium and zinc soaps of DFA derived from direct neutralization method and metathesis process, respectively

result suggests that there might be a little unreacted $\mathrm{Ca}(\mathrm{OH})_{2}$ in C22TA-Ca-I and $\mathrm{ZnO}$ in the C22TA-Zn-I thermal stabilizers. In contrast, the measured $\mathrm{Ca}$ and $\mathrm{Zn}$ contents in C22TA-Ca-II and C22TA-Zn-II were $10.60 \%$ and $15.22 \%$, respectively, which suggest the conversions of the carboxylic acid groups were $92.5 \%$ and $87.7 \%$, respectively.The FTIR and the metal content of calcium or zinc soap suggest that MAME were successfully converted to calcium and zinc soaps by direct neutralization and metathesis process, respectively. However, there was still a small fraction of the carboxylic acid groups, which was not converted into calcium or zinc soaps.

\section{Effects of the thermal stabilizers of DFA on the thermal stability of PVC}

PVC processing stabilization can be gauged by the induction period and stabilization period. The induction time is the time of the early stage of the irreversible color change measured under specified practical conditions and a certain temperature. The stability time is defined as the time from begining to the specified point at which the irreversible changes have made the compound no longer reclaimable or begins to endanger the processing equipment. Both the induction time and stability time can represent the PVC stability. For comparison, all PVC compounds were formulated on the same basis in terms of individual ingredients (Table 1). 


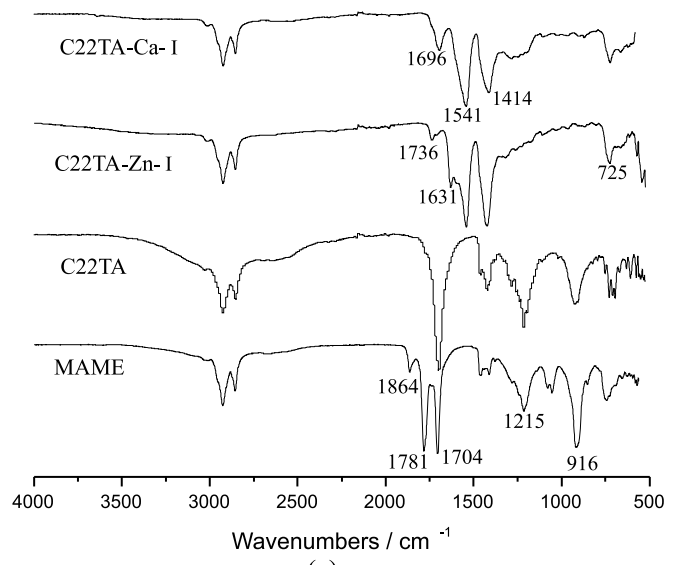

(a)

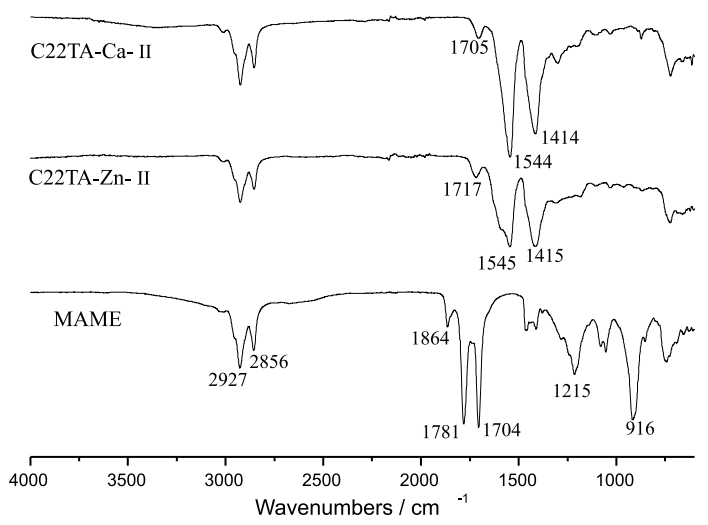

(b)

Figure 2. FTIR spectra of MAME, C22TA, C22TA-Ca and C22TA-Zn. (a) and (b) were zinc tricarboxylates and calcium tricarboxylates derived from direct neutralization method and metathesis process, respectively

Table 1. Formulations for three thermal stabilizers

\begin{tabular}{|l|c|c|c|}
\hline \multirow{2}{*}{ Ingredients } & \multicolumn{2}{c|}{ Formulations } \\
\cline { 2 - 4 } & I & II & III \\
\hline PVC & 100 & 100 & 100 \\
\hline DOTP & 50 & 50 & 50 \\
\hline CaSt $_{2}+$ ZnSt $_{2}$ & $2.4 \mathrm{~g}+0.6 \mathrm{~g}$ & 0 & 0 \\
\hline DFA-Ca-I+ DFA-Zn-I & 0 & $2.4 \mathrm{~g}+0.6 \mathrm{~g}$ & 0 \\
\hline DFA-Ca-II + DFA-Zn-II & 0 & 0 & $2.4 \mathrm{~g}+0.6 \mathrm{~g}$ \\
\hline
\end{tabular}

The static thermal stability times $\left(T_{\mathrm{ss}}\right)$ were measured using Congo Red test. The effects of each stabilizer on the $T_{\mathrm{ss}}$ of the $\mathrm{PVC}$ compounds at $185^{\circ} \mathrm{C}$ are shown in Table 2. The results indicated that the DFA-Ca-I /DFA-Zn-I significantly influenced long-term heat stability. The $T_{\text {ss }}$ values of the formulations followed the order of DFA-Ca-I /DFA-Zn-I (49.8 min)> DFA-Ca-II /DFA-Zn-II (31.0 min) $>\mathrm{CaSt}_{2} / \mathrm{ZnSt}_{2}(20.9 \mathrm{~min})$.

Discoloration test results for the thermal stabilizers (at $185^{\circ} \mathrm{C}$ ) are also shown in Table 2. The PVC strips containing the $\mathrm{CaSt}_{2} / \mathrm{ZnSt}_{2}$ stabilizer exhibited excellent early color retention (up to $10 \mathrm{~min}$ ), but turned competely black in $30 \mathrm{~min}$. The PVC strips with the DFA-Ca-I/DFA-Zn-I stabilizer lasted $60 \mathrm{~min}$ before turning completely black. The PVC samples stabilized by the DFA-Ca-I/ DFA-Zn-I stabilizer had longer stability times than those using DFA-Ca-II/DFA-Zn-II and $\mathrm{CaSt}_{2} / \mathrm{ZnSt}_{2}$. According to the $\mathrm{Ca}$ and $\mathrm{Zn}$ contents in DFA-Ca-I/DFA-Zn-I and DFA-Ca-II/DFA-Zn-II, it can be concluded that the conversions of the carboxylic acid groups in DFA-Ca-I/ DFA-Zn-I were slightly lower than DFA-Ca-II/DFA-Zn-II. However, the thermal stability of DFA-Ca-I/DFA-Zn-I is comparable with DFA-Ca-II/DFA-Zn-II thermal stabilizer. This result might be due to the slightly higher zinc content in DFA-Zn-II than DFA-Zn-I. As we know, zinc soaps can effectively inhibit discoloration by substituting labile chlorine atoms in PVC chains but provide a poor long-term stability because of the phenomenon of "zinc burn"16-18. In spite of the zinc content of DFA$\mathrm{Zn}$-II can be turn down by decreasing the mole ratio of $\mathrm{ZnSO}_{4} \cdot 7 \mathrm{H}_{2} \mathrm{O}$ to DFA in the process of synthesizing DFA-Zn-II. However, the process of synthesizing DFA$\mathrm{Zn}$-II resulted in a lot of waste water.

The TGA and DTG curves for PVC with each of the thermal stabilizers are shown in Figure 3. The kinetic parameters for the three thermal stabilizers are summarized in Figure 4. PVC compositions stabilized with DFA-Ca-I/DFA-Zn-I had higher thermal degradation temperatures than PVC stabilized with the $\mathrm{CaSt}_{2} / \mathrm{ZnSt}_{2}$ and DFA-Ca- -II/DFA-Zn-II. The $E_{a}$ values of the formulations followed the order of DFA-Ca-I/DFA-Zn-I (115.1 $\left.\mathrm{kJ} \cdot \mathrm{mol}^{-1}\right)>$ DFA-Ca-II/DFA-Zn-II $\left(109.7 \mathrm{~kJ} \cdot \mathrm{mol}^{-1}\right)$ $>\mathrm{CaSt}_{2} / \mathrm{ZnSt}_{2}\left(101.4 \mathrm{~kJ} \cdot \mathrm{mol}^{-1}\right)$. The PVC sample containing DFA-Ca-I/DFA-Zn-I had the highest thermal degradation activation energy $\left(E_{\mathrm{a}}\right)$. Generally, a higher $E$ suggests a higher resistance to thermal degradation. Therefore, the TGA results indicate that DFA-Ca-I/DFA-Zn-I was a more effective PVC thermal stabilizer than either the commercial thermal stabilizer or DFA-Ca-II/ DFA-Zn-II thermal stabilizer.

Table 2. Congo Red tests and discoloration tests of PVC samples in the presence of various stabilizers at $185^{\circ} \mathrm{C}$

\begin{tabular}{|l|c|c|c|c|c|c|c|c|}
\hline \multicolumn{1}{|c|}{ Formulations } & \multirow{2}{*}{$T_{\text {ss }}[\mathrm{min}]$} & \multicolumn{5}{c|}{ Degradation time [min] } \\
\cline { 4 - 9 } & & 0 & 10 & 20 & 30 & 40 & 50 & 60 \\
\hline $\mathrm{CaSt}_{2} / \mathrm{ZnSt}_{2}$ & 20.9 & & & & & & \\
\hline DFA-Ca-I/DFA-Zn-I & 49.8 & & & & & & & \\
\hline DFA-Ca-II/DFA-Zn-II & 31.0 & & & & & & & \\
\hline
\end{tabular}



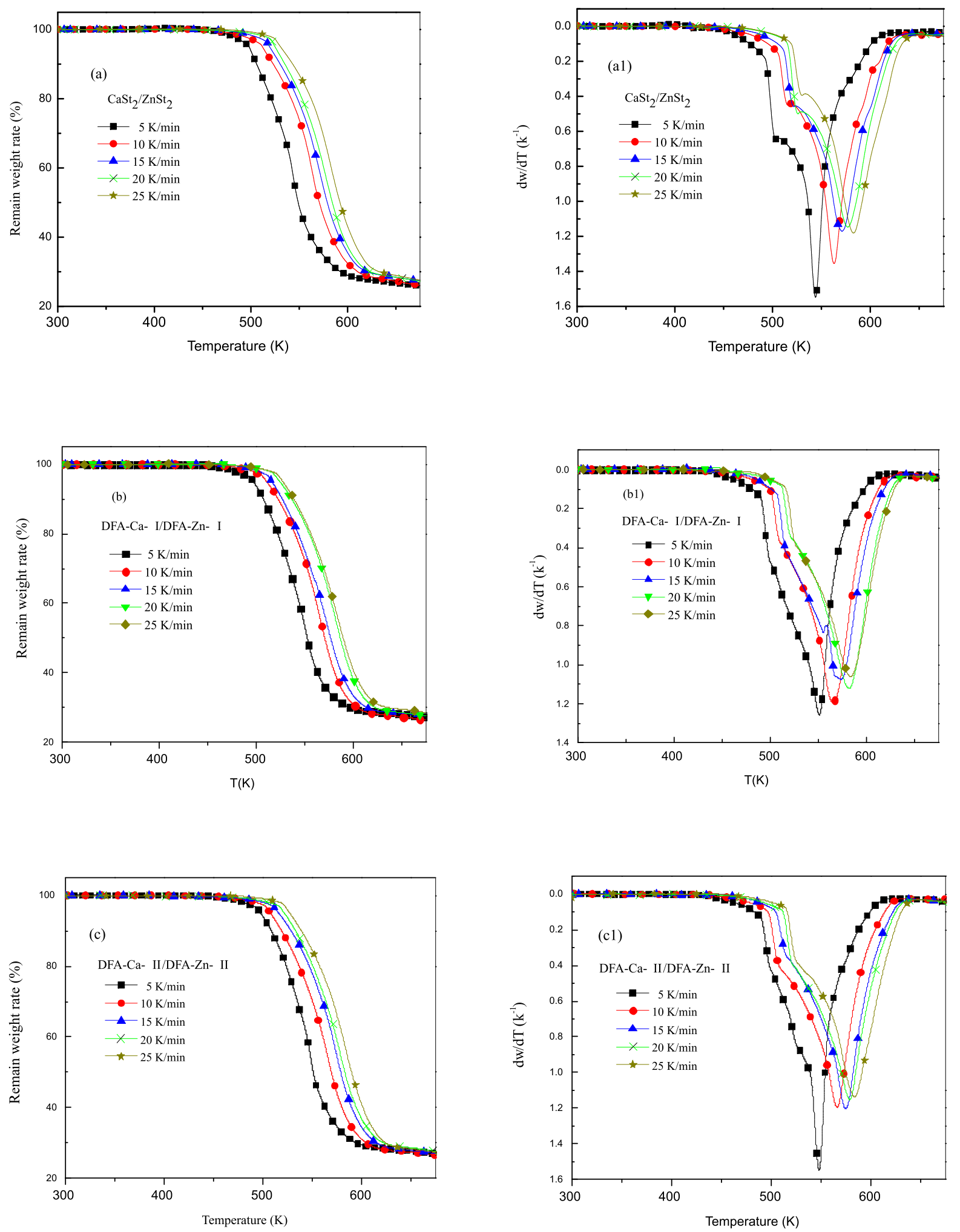

Figure 3. TG and DTG curves of the stabilized PVC samples at various heating rates

Effects of the thermal stabilizers of C22TA on the thermal stability and mechanical properties of PVC

All PVC compounds stabilized with the thermal stabilizers of C22TA were formulated on the same basis in terms of individual ingredients (Table 3). DOP is a plasticizer and epoxidized soybean oil (ESO) is an auxiliary plasticizer with heat stabilizing effect. The epoxy groups of ESO can easily react with the $\mathrm{HCl}$ and hence prevents the autoacceleration. Zinc soaps can effectively inhibit initial discoloration by substituting labile chlorine atoms in PVC chains but provide a poor long-term stability because of the generated $\mathrm{ZnCl}_{2}$ which is a catalyst for the elimination of $\mathrm{HCl}$. $\beta$-diketone (DK) is an auxiliary thermal stabilizer, which can effectively improve initial color and the long-term stability of the thermal stabilizers $^{29-30}$. The functional mechanism of DK as an auxiliary thermal stabilizer is shown in Scheme 2.

Effects of the thermal stabilizers of C22TA on the thermal stability of PVC

Dynamic thermal stability time $\left(T_{d s}\right)$ and equilibrium torque of the four films (Table 4) were measured using a Haake torque rheometer at $180^{\circ} \mathrm{C}$ (Fig. 5), while static thermal stability time $\left(T_{s s}\right)$ were measured using 


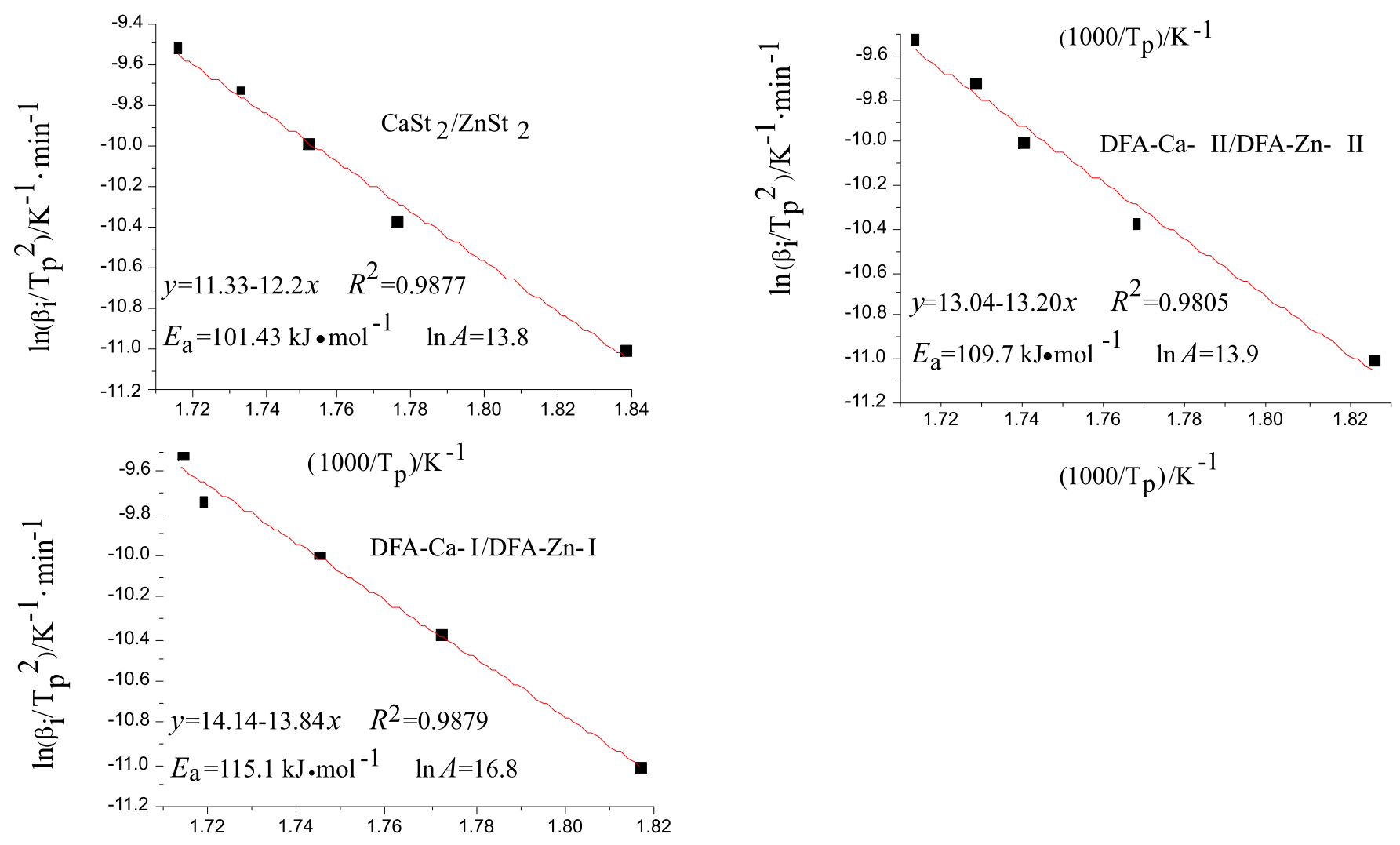

Figure 4. Plots of $\ln \left(\beta \mathrm{i} / \mathrm{Tp}^{2}\right)$ versus $1000 / \mathrm{Tp}$ and kinetic parameters for the PVC samples stabilized with four thermal stabilizers

a Congo red test device at $185^{\circ} \mathrm{C}$. The results of $T_{s s}, T_{d s}$ and the equilibrium torque are summarized in Table 4. The $T_{s s}$ values of different PVC compounds followed the order of C22TA-Ca/Zn Stab-I/ESO/DK (51.8 min) $>$ C22TA-Ca/Zn Stab-II/ESO/DK (45.8 min) > C22TA-Ca/Zn Stab-I (27.2 min) > C22TA-Ca/Zn Stab-II (17.8 min). This result is consistent with the $T_{d s}$ result. The stabilizers prepared by direct neutralization exhibited higher thermal stabilizing effect for PVC compounds, and addition of ESO and DK greatly improved thermal stability of the PVCcompounds. The equilibrium torque values of different PVC compounds followed the order of C22TA-Ca/Zn Stab-II (3.5 Nm) > C22TA-Ca/Zn Stab-I (3.2 Nm) > C22TA-Ca/Zn Stab-I/ESO/DK (2.6 Nm) > C22TA-Ca/Zn Stab-II/ESO/DK (2.4 Nm). In general, the stabilizers prepared by direct neutralization led to high equilibrium torque than the stabilizers prepared by metathesis process, and addition of ESO and DK lowered the equilibrium torque. Because the melt viscosity is proportional to the torque, lower equilibrium torque suggests a more optimal processing rheology. These results also indicate that ESO and DK were more effective

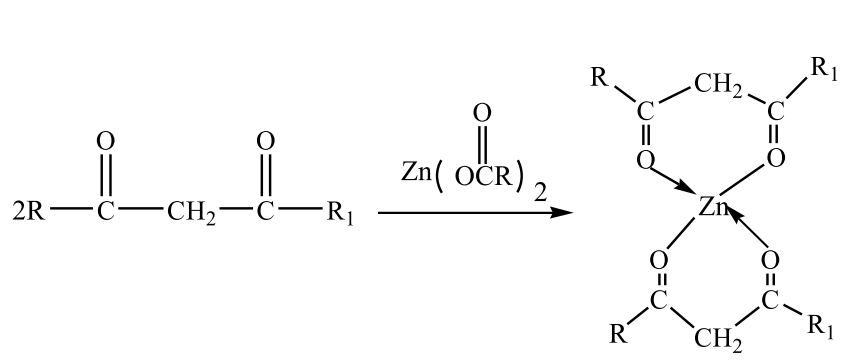<smiles>CC=CC(Cl)CC(C)Cl</smiles><smiles>[R]C(=O)C=C([R])OC(C=CCOC(=O)OCC(=O)OC(C)(Cl)C(Cl)Cl)CC(C)Cl</smiles>

Scheme 2. Functional mechanism of $\beta$-diketone as an auxiliary PVC thermal stabilizer

Table 3. Formulations of PVC samples stabilized by different thermal stabilizers

\begin{tabular}{|l|c|c|c|c|}
\hline \multirow{2}{*}{\begin{tabular}{l} 
Ingredients \\
\cline { 2 - 5 }
\end{tabular}} & Formulation I & Formulation II & Formulation III & Formulation IV \\
\cline { 2 - 5 } & C22TA-Ca/Zn-Stab-II & C22TA-Ca/Zn-Stab-I & $\begin{array}{c}\text { C22TA-Ca/Zn- } \\
\text {-Stab-II/ESO/DK }\end{array}$ & $\begin{array}{c}\text { C22TA-Ca/Zn-Stab- } \\
\text {-I/ESO/DK }\end{array}$ \\
\hline PVC & 100 & 100 & 100 & 100 \\
\hline DOP & 50 & 50 & 50 & 50 \\
\hline C22TA-Ca-II & 1.6 & 0 & 1.6 & 0 \\
\hline C22TA-Zn-II & 0.4 & 0 & 0.4 & 1.6 \\
\hline C22TA-Ca-I & 0 & 1.6 & 0 & 0.4 \\
\hline C22TA-Zn-I & 0 & 0.4 & 3 & 3 \\
\hline Epoxidized soy oil (ESO) & 0 & 0 & 0.3 & 0.3 \\
\hline$\beta$-diketone (DK) & 0 & 0 & 0.3 & \\
\hline
\end{tabular}


Table 4. Comparison of effects of different thermal stabilizers on thermal stability and processing rheological property of PVC compounds

\begin{tabular}{|l|c|c|c|c|}
\hline Sample entry & Stabilizer & $T_{\mathrm{ds}}{ }^{\mathrm{a}} /\left[180^{\circ} \mathrm{C}\right]$ & $T_{\mathrm{ss}}{ }^{\mathrm{b}} /\left[185^{\circ} \mathrm{C}\right]$ & Equilibrium torque/Nm \\
\hline 1 & C22TA-Ca/Zn Stab-II & $16.8 \mathrm{~min}$ & $17.8 \mathrm{~min}$ & 3.5 \\
\hline 2 & C22TA-Ca/Zn Stab-II/ESO/DK & $38.1 \mathrm{~min}$ & $45.8 \mathrm{~min}$ & 2.4 \\
\hline 3 & C22TA-Ca/Zn Stab-I & $20.7 \mathrm{~min}$ & $27.2 \mathrm{~min}$ & 3.2 \\
\hline 4 & C22TA-Ca/Zn Stab-I/ESO/DK & $40.0 \mathrm{~min}$ & $51.8 \mathrm{~min}$ & 2.6 \\
\hline
\end{tabular}

${ }^{a}$ The dynamic thermal stability time; ${ }^{b}$ Static thermal stability time.

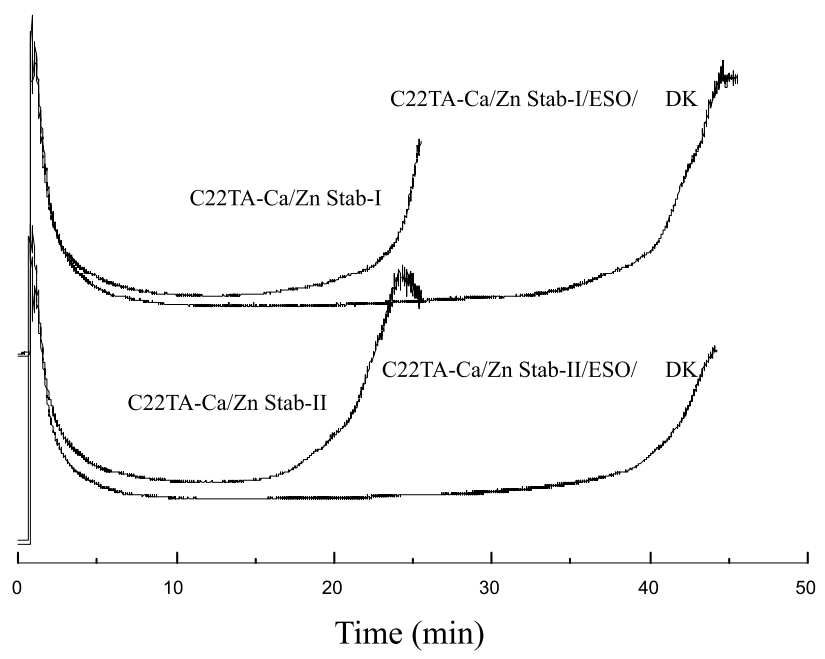

Figure 5. Dynamic thermal stability times of the PVC compounds stabilized with different thermal stabilizers at $180^{\circ} \mathrm{C}$

in improving the processing rheological properties and stabilizing PVC.

Table 5 shows the results of discoloration tests on the strips of PVC compounds containing different thermal stabilizers at $185^{\circ} \mathrm{C}$. As a result, the long-term stability of different PVC compounds followed the order of C22TA-Ca/Zn Stab-I/ESO/DK > C22TA-Ca/Zn Stab-II/ESO/DK $>$ C22TA-Ca/Zn Stab-I > C22TA-Ca/Zn Stab-II. The PVC strip of C22TA-Ca/Zn Stab-I/ESO/DK presented the lightest color after $40 \mathrm{~min}$ and required the longest time (80 $\mathrm{min}$ ) to turn completely black. Again, PVC stabilized by C22TA-Ca/Zn Stab-I displayed more excellent thermal stability than that stabilized by C22TA-Ca/Zn Stab-II. This result might be due to a small amount of the unreacted $\mathrm{Ca}(\mathrm{OH})_{2}$ and $\mathrm{ZnO}$ in the $\mathrm{C} 22 \mathrm{TA}-\mathrm{Ca} / \mathrm{Zn}$ Stab-I thermal stabilizers. It was very hard to remove the unreacted $\mathrm{Ca}(\mathrm{OH})_{2}$ and $\mathrm{ZnO}$ from the $\mathrm{C} 22 \mathrm{TA}-\mathrm{Ca} /$ $\mathrm{Zn}$ Stab-I thermal stabilizers. However, the $\mathrm{Ca}(\mathrm{OH})_{2}$ was converted to $\mathrm{CaO}$ during heating, which then neutralized the $\mathrm{HCl}$ evolved during dehydrochlorination. The little
$\mathrm{ZnO}$ in C22TA-Zn-I also captured the $\mathrm{HCl}$ evolved and subsequently prevented accelerated dehydrochlorination to some extent. Compared with C22TA-Ca/Zn Stab-II thermal stabilizers, the higher thermal stability could be attributed to the dual action of the metal carboxylate and hydroxide present in C22TA-Ca/Zn Stab-I thermal stabilizers. That does not means that a large amount of $\mathrm{Ca}(\mathrm{OH})_{2}$ and $\mathrm{ZnO}$ in thermal stabilizers will not influence the thermal stability. For instance, excess $\mathrm{ZnO}$ can cause the phenomenon of "zinc burn" due to the existence of excess $\mathrm{ZnCl}_{2}$. These results of the effects of the mixed $\mathrm{Ca} / \mathrm{Zn}$ thermal stabilizers derived from C22TA and DFA on PVC thermal stability indicated that the direct neutralization process not only had the advantages of simple process, short production cycle and no waste water, but could offer excellent mixed $\mathrm{Ca} / \mathrm{Zn}$ thermal stabilizers for PVC when compared with the metathesis process. On the other hand, a small amount of the unreacted $\mathrm{Ca}(\mathrm{OH})_{2}$ and $\mathrm{ZnO}$ brought by the direct neutralization process did not destory the thermal stabilizing effect of the mixed $\mathrm{Ca} / \mathrm{Zn}$ thermal stabilizers.

Effects of the thermal stabilizers of C22TA on the mechanical properties of PVC

Figure 6 shows the changes of storage modulus $\left(E^{\prime}\right)$ and damping $(\tan \delta)$ of different PVC film samples with temperature. All compositions displayed very similar $E^{\prime}$ in the glassy state and glass transition region. The PVC compounds experienced a sharp drop in the broad glass transition region from -30 to $40^{\circ} \mathrm{C}$. Above $30^{\circ} \mathrm{C}$, the $E^{\prime}$ of the C22TA-Ca/Zn Stab-I/ESO/DK stabilized PVC was slightly higher than others, and the one with C22TA-Ca/ $\mathrm{Zn}$ Stab-I exhibited lower storage modulus. In general, $E$, changed little from stabilizer to stabilizer. On the other hand, all PVC compounds exhibited a single tan $\delta$ peak, indicating a homogeneous morphological structure for all compounds. If the peak temperature of $\tan \delta$ peak is assumed to be $T_{g}$, the broad glass transition region seen in all these compounds is a typical phenomenon for pla-

Table 5. Discoloration of PVC samples as a function of degradation time at $185^{\circ} \mathrm{C}$

\begin{tabular}{|c|c|c|c|c|c|c|c|c|c|}
\hline \multirow{2}{*}{ Formulations } & \multicolumn{9}{|c|}{ Time [min] } \\
\hline & 0 & 10 & 20 & 30 & 40 & 50 & 60 & 70 & 80 \\
\hline C22TA-Ca/Zn Stab-II & & & & & & & & & \\
\hline C22TA-Ca/Zn Stab-I & & & & & & & & & \\
\hline C22TA-Ca/Zn Stab-II + ESO/DK & & & & & & & & & \\
\hline C22TA-Ca/Zn Stab-I + ESO/DK & & & & & & & & & \\
\hline
\end{tabular}



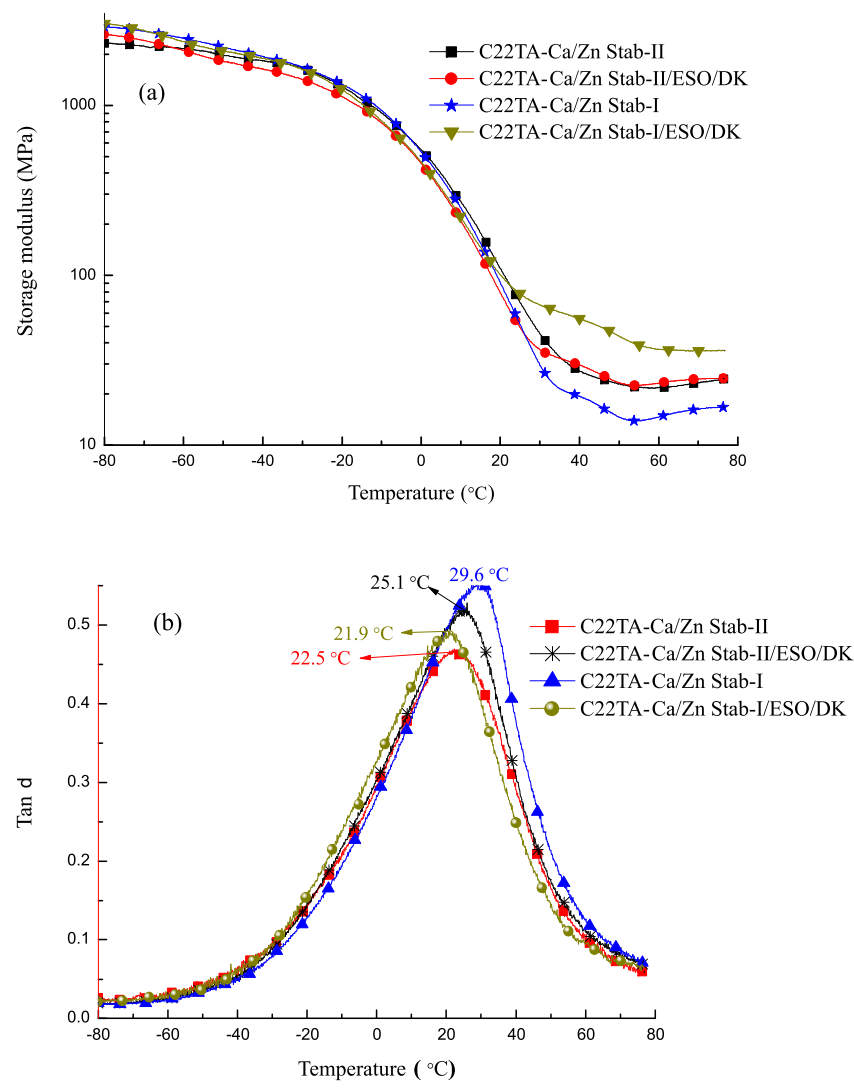

Figure 6. Storage modulus (E') and $\tan \delta$ of the PVC samples with different thermal stabilizers

sticized polymers with different additives and is a result of multiple relaxation times of the polymer molecules.

\section{CONCLUSIONS}

Two technologies of direct neutralization and metathesis methods were used to synthesize zinc and calcium salts of DFA and MAME. Results indicated that PVC films stabilized by the thermal stabilizers produced using direct neutralization method (DFA-Ca-I/DFA-Zn-I) offered greater thermal stability than the metathesis products (DFA-Ca-II/DFA-Zn-II). PVC films stabilized by C22TA- $\mathrm{Ca} / \mathrm{Zn}$ Stab-I thermal stabilizer have better processing rheological property and thermal stability than that of C22TA-Ca/Zn Stab-II. For example, the $E_{a}$ values of the formulations followed the order of DFA-Ca-I / DFA-Zn-I $\left(115.1 \mathrm{~kJ} \cdot \mathrm{mol}^{-1}\right)>$ DFA-Ca-II/DFA-Zn-II $\left(109.7 \mathrm{~kJ} \cdot \mathrm{mol}^{-1}\right)>\mathrm{CaSt}_{2} / \mathrm{ZnSt}_{2}\left(101.4 \mathrm{~kJ} \cdot \mathrm{mol}^{-1}\right)$. The static stability time values at $185^{\circ} \mathrm{C}$ of different $\mathrm{PVC}$ compounds followed the order of C22TA-Ca/Zn Stab-I/ESO/ $\beta$-diketone $(51.8 \mathrm{~min})>\mathrm{C} 22 \mathrm{TA}-\mathrm{Ca} / \mathrm{Zn} \mathrm{Stab}-\mathrm{II} /$ $\mathrm{ESO} / \beta$-diketone (45.8 min) > C22TA-Ca/Zn Stab-I (27.2 $\mathrm{min})>\mathrm{C} 22 \mathrm{TA}-\mathrm{Ca} / \mathrm{Zn} \mathrm{Stab}-\mathrm{II}$ (17.8 min). This study suggests that the raw materials of this research are renewable resources. This study will be beneficial to promote the domestic low-carbon economic development and find the properties difference between the two kinds of synthetic technology of the mixed $\mathrm{Ca} / \mathrm{Zn}$ thermal stabilizers.

\section{ACKNOWLEDGEMENTS}

The authors are grateful to the Jiangsu Province Biomass Energy and Materials Laboratory for financial support, China (grant number: JSBEM-S-201508).

\section{LITERATURE CITED}

1. Nagy, T.T., Kelen, T., Turcsányi, B. \& Tüdös, F. (1980). The reinitiation mechanism of $\mathrm{HCl}$ catalysis in $\mathrm{PVC}$ degradation. Polym. Bull. 2(1), 77-82. DOI: 10.1007/BF00275557.

2. Braun, D. (1981). Thermal degradation of poly(vinyl chloride), in Development in polymer degradation, Grassie, N., Eds.; Appl. Sci. Publ.: London, pp 101.

3. Vrandečić, N.S., Klarić, I. \& Roje, U. (2001). Effect of $\mathrm{Ca} / \mathrm{Zn}$ stabiliser on thermal degradation of poly(vinyl chloride)/ chlorinated polyethylene blends. Polym. Degrad. Stab. 74(2), 203-212. http://dx.doi.org/10.1016/S0141-3910(01)00013-1.

4. Benavides, R., Castillo, B.M., Castañeda, A.O., López, G.M. \& Arias, G. (2001). Different thermo-oxidative degradation routes in poly(vinyl chloride). Polym. Degrad. Stab. 73(1), 417-423. http://dx.doi.org/10.1016/S0141-3910(01)00122-7

5. Benaniba, M.T., Belhaneche-Bensemra, N. \& Gelbard, G. (2003). Stabilization of PVC by epoxidized sunflower oil in the presence of zinc and calcium stearates. Polym. Degrad. Stab. 82(2), 245-249. http://dx.doi.org/10.1016/S0141-3910(03)00178-2

6. Lin, Y.J., Wang, J.R., Evans, D.G. \& Li, D.Q. (2006). Layered and intercalated hydrotalcite-like materials as thermal stabilizers in PVC resin. J. Phys. Chem. Sol. 67(5-6), 998-1001. http://dx.doi.org/10.1016/j.jpcs.2006.01.016

7. Bao, Y., Huang, Z., Li, S. \& Weng, Z. (2008). Thermal stability, smoke emission and mechanical properties of poly(vinyl chloride)/hydrotalcite nanocomposites. Polym. Degrad. Stab. 93(2), 448-455. http://dx.doi.org/10.1016/j.polymdegradstab.2007.11.014

8. Naqvi, M.K., Unnikrishnan, P.A., Sharma, Y.N. \& Bhardwaj, I.S. (1984). Effect of calcium and zinc carboxylates on the thermal stabilisation of PVC. Eur. Polym. J. 20(1), 95-98. DOI: 10.1016/0014-3057(84)90231-3.

9. Gökçel, H.I., Balköse, D. \& Köktürk, U. (1999). Effects of mixed metal stearates on thermal stability of rigid PVC. Eur. Polym. J. 35(8), 1501-1508. http://dx.doi.org/10.1016/ S0014-3057(98)00217-1

10. Farone, E.V., Labovitz, B.P. \& Kruse, N.A. (2013). Process for preparing liquid overbased metal carboxylates, mixed metal stabilizers containing same, and stabilized halogen-containing polymers therewith. U.S. Patent 2013137806(A1).

11. Fang, L., Song, Y.H., Zhu, X.N. \& Zheng, Q. (2009). Influence of lanthanum stearate as a co-stabilizer on stabilization efficiency of calcium/zinc stabilizers to polyvinyl chloride. Polym. Degrad. Stab. 94(5), 845-850. http://dx.doi.org/10.1016/j. polymdegradstab.2009.01.024

12. Razvan, C., Beck, R., Kurzinger, A., Purzer, A.W. \& Rosentha, M. (1991). Basic calcium-aluminium-hydroxy phosphites, process for their manufacture and use. D.E. Patent $3941902 \mathrm{Cl}$.

13. Wang, M., Xu, J.Y., Wu, H. \& Guo, S.Y. (2006). Effect of pentaerythritol and organic tin with calcium/zinc stearates on the stabilization of poly(vinyl chloride). Polym. Degrad. Stab. 91(9), 2101-2109. http://dx.doi.org/10.1016/j.polymdegradstab.2006.01.011

14. Kalouskova, R., Novotna, M. \& Vymazal, Z. (2004). Investigation of thermal stabilization of poly(vinyl chloride) by lead stearate and its combination with synthetic hydrotalcite. Polym. Degrad. Stab. 85(2), 903-909. http://dx.doi.org/10.1016/j. polymdegradstab.2004.04.008

15. Edenbaum, J. (1992). Plastics additives and modifiers handbook, in Mixed-metal stabilizers for polyvinyl chloride; Verbiar, L., Eds.;. Van Nostrand Reinhold Compary: New York, pp 272-296.

16. Folarin, O.M. \& Sadiku, E.R. (2011). Thermal stabilizers for poly(vinyl chloride): A review. Int. J. Phys. Sci. 6(18), 4323-4330. http://www.academicjournals.org/IJPS

17. Abbås, K.B. \& Sörvik, E.M. (1980). Heat stabilizers for poly(vinyl chloride). I. Synergistic systems based on calcium/ zinc stearate. J.Vinyl Addit. Technol. 2(2), 87-94. DOI: 10.1002/ vnl.730020205. 
18. Manzoor, W., Yousaf, S.M. \& Ahmad, Z. (1996). Degradation of PVC: Effect of zinc chloride on the concentration of polyenes. Polym. Degrad. Stab. 51(3), 295-299. DOI: 10.1016/0141-3910(95)00186-7.

19. Balköse, D., Gökçel, H.I. \& Göktepe, S.E. (2001). Synergism of $\mathrm{Ca} / \mathrm{Zn}$ soaps in poly(vinyl chloride) thermal stability. Eur. Polym. J. 37(6), 1191-1197. http://dx.doi.org/10.1016/ S0014-3057(00)00233-0

20. Lévai, G., Ocskay, G. \& Nyitrai, Z. (1989). Kinetics of the stabilizing effect of calcium and zinc stearates in the thermal degradation of PVC: part II. Polym. Degrad. Stab. 25(1), 61-72. DOI: 10.1016/0141-3910(89)90024-4.

21. Liu, Y.B., Liu, W.Q. \& Hou, M.H. (2007). Metal dicarboxylates as thermal stabilizers for PVC. Polym. Degrad. Stab. 92(8), 1565-1571. http://dx.doi.org/10.1016/j.polymdegradstab.2007.05.003

22. Ree, M., Bae, J.Y., Jung, J.H. \& Shin, T.J. (1999). A new copolymerization process leading to poly(propylene carbonate) with a highly enhanced yield from carbon dioxide and propylene oxide. J. Polym. Sci. Part A Polym. Chem. 37(12), 1863-1876. DOI: $10.1002 /($ SICI)1099-0518(19990615)37:12<1863::AID-POLA16>3.0.CO;2-K.

23. Wang, M., Xia, J., Jiang, J., Li, S., Huang, K., Mao, W. \& Li, M. (2016). A novel liquid $\mathrm{Ca} / \mathrm{Zn}$ thermal stabilizer synthesized from tung-maleic anhydride and its effects on thermal stability and mechanical properties of PVC. Polym. Degrad. Stab. 133, 136-143. http://dx.doi.org/10.1016/j.polymdegradstab.2016.08.010

24. Guo, Y., Zheng, Y.Y., Qiu, S.C., Zeng, A.R. \& Li, B.M. (2011). Metal lanolin fatty acid as novel thermal stabilizers for rigid poly(vinyl chloride). J. Rare Earth. 29(5), 401-406. DOI: 10.1016/S1002-0721(10)60468-1.

25. Wang, M., Xia, J., Jiang, J., Li, S. \& Li, M. (2016). Mixed calcium and zinc salts of N-(3-aminobenzoic acid)terpene-maleamic acid: preparation and its application as novel thermal stabilizer for poly(vinyl chloride). RSC Adv. 6(99), 97036-97047. DOI: 10.1039/C6RA19523G.

26. Li, M., Zhang, J., Xin, J., Huang, K., Li, S., Wang, M. \& Xia, J. (2016). Design of green zinc-based thermal stabilizers derived from tung oil fatty acid and study of thermal stabilization for PVC. J. Appl. Polym. Sci. DOI: 10.1002/app.44679.

27. Yoshizawa, F., Kikuchi, F., Kojima, S. \& Yuasa, K. (1992). Continuous process for preparing metallic soaps. U.S. Patent 5175322.

28. Pietralla, N., Ausserbauer, V. \& Rosenthal, C. (1981). Method for the production of metal soaps. U.S. Patent 4294771.

29. Bensemra, N., Hoang, T.V., Guyot, A., Gay, M. \& Carette, L. (1989). Thermal dehydrochlorination and stabilization of poly(vinylchloride) in solution: Part IV-Synergistic effects of $\beta$-diketone compouds and metal soap stabilizers. Polym. Degrad. Stab. 24(2), 89-111. DOI: 10.1016/0141-3910(89)90105-5.

30. Michel, A., Hoang, T.V., Perrin, B. \& Llauro, M.F. (1981). Synergistic mechanisms of $\beta$-diketone derivatives and zinc-calcium soaps in PVC stabilisation. Polym. Degrad. Stab. 3(3), 107-119. DOI: 10.1016/0141-3910(81)90003-3. 